\title{
Transjugular intrahepatic portosystemic shunt (TIPS) creation prior to abdominal operation: a retrospective analysis
}

\author{
Adam Schmitz, Paul Haste, MD, Matthew S. Johnson, MD \\ Indiana University School of Medicine, Department of Radiology, 550 N University Blvd \\ Indianapolis, IN 46202
}

\begin{abstract}
Purpose: Transjugular intrahepatic portosystemic shunt (TIPS) creation is most commonly performed for patients with refractory ascites or variceal hemorrhage. While TIPS have also been created prior to planned abdominal operation to decrease morbidity related to portal hypertension, there are limited data supporting its effectiveness in that indication. The goal of this study was to determine if preoperative TIPS creation allows for successful abdominal operation with limited morbidity.
\end{abstract}

Methods: A retrospective review of records of 22 consecutive patients who underwent TIPS creation for the specific indication of improving surgical candidacy, between 2011 and 2016, was performed. Clinical and serologic data were obtained for 21 patients (one patient was excluded since she was completely lost to follow up after TIPS creation). The primary endpoint was whether patients underwent planned abdominal operation following TIPS. Operative outcomes and reasons that patients failed to undergo planned operation were examined as secondary endpoints. The mean age was $56.4 \pm 8.8$ years, and the mean Child-Pugh and Model for End-Stage Liver Disease (MELD) scores were $7.2 \pm 1.5$ and $11.9 \pm 4.3$, respectively.

Results: TIPS creation was performed in all 21 patients with a thirty-day mortality rate of $9.5 \%$. Eleven patients (52.4\%) subsequently underwent abdominal operation after which the thirty-day postoperative mortality rate was $0 \%$. One patient $(9.1 \%)$ had major perioperative morbidity related to portal hypertension and presented with surgical wound dehiscence and infection requiring drain placement and antibiotic therapy.

Conclusions: In this population, TIPS allowed successful abdominal operation in the majority of patients, with thirty-day TIPS mortality of $9.5 \%$, no perioperative mortality, and $9.1 \%$ major postoperative morbidity attributable to portal hypertension.

awschmit@iupui.edu.

Authorship

Adam Schmitz made substantial contributions to the design of this study, analyzed the data, and wrote and approved the final manuscript form. In addition Mr. Schmitz agrees to be accountable for the information presented in this manuscript.

Dr. Paul Haste made substantial contributions to the design of this study, aided in the drafting process, and approved the final manuscript form. In addition Dr. Haste agrees to be accountable for the information presented in this manuscript.

Dr. Matthew S. Johnson made substantial contributions to the design of this study, aided in the drafting process, and approved the final manuscript form. In addition Dr. Johnson agrees to be accountable for the information presented in this manuscript.

Conflicts of Interest

Adam Schmitz: None declared

Dr. Paul Haste: None declared

Dr. Matthew S. Johnson: Dr. Johnson is a paid consultant to Cook Medical 


\section{Keywords}

Transjugular intrahepatic portosystemic shunt; TIPS; surgery; portal hypertension

\section{Introduction}

Transjugular intrahepatic portosystemic shunt (TIPS) creation is most commonly performed for one of two indications: variceal hemorrhage or refractory ascites [1]. A third indication that may lead to TIPS creation is portal decompression prior to planned abdominal operation. Cirrhosis is a widely recognized predictor of operative morbidity and mortality, with a recent systematic review indicating that cirrhotic patients undergoing any surgical procedure have postoperative morbidity and thirty-day mortality rates of $30.1 \%$ and $11.6 \%$, respectively [2]. Patients with cirrhosis and concomitant portal hypertension have even greater operative risks, which can be accurately assessed by Child-Pugh and model for endstage liver disease (MELD) scores [2,3]. One recent study found that patients with portal hypertension undergoing gastrointestinal surgery had a 6-fold increase in 30-day mortality rates compared to patients without portal hypertension [4]. Some studies have indicated that portal decompression via neoadjuvant TIPS can ameliorate operative risks and improve outcomes, while others describe no benefit [5, 6, 7]. Definitive answers have been difficult to pinpoint due to the relative infrequency of this indication for TIPS and the small sample sizes in the published literature. In addition, the practicality of using TIPS to facilitate abdominal operation has yet to be examined in the United States where non-alcoholic fatty liver disease (NAFLD) is the most common cause of liver disease [8]. The goal of this study was to determine the percentage of patients who underwent abdominal operation following preoperative TIPS creation and to understand the relationship between preoperative TIPS and perioperative outcomes.

\section{Materials and Methods}

This retrospective study was conducted at a single university medical center, was HIPAA compliant and approved by the institutional review board. Patients who underwent TIPS creation between 2011-2016 were identified through a database search, and these patients were further stratified by indication for TIPS. Twenty-two patients underwent TIPS creation with the specific goal of improving surgical candidacy. One patient for whom no follow up data were available was excluded, yielding a final cohort of 21 patients.

Clinical and serologic data were collected for all patients. Patient demographics, liver disease etiology, laboratory values, and physiologic measurements were recorded. Medical history including the presence of varices, ascites, and encephalopathy was also taken into consideration. Liver function was assessed using MELD and Child-Pugh scores. Clinical and serologic data for all patients prior to TIPS is summarized in Table 1.

All patients had manifestations of portal hypertension prior to TIPS (varices, ascites, or both). Patients were referred for TIPS creation specifically to improve their surgical candidacy through decompression of varices $(n=11)$ or reduction of ascites $(n=10)$. Seven of these patients did not have a history of variceal bleeding, but rather had varices noted on pre- 
operative imaging. The planned abdominal operations included hernia repair $(n=10)$, sleeve gastrectomy $(n=6)$, cholecystectomy $(n=1)$, gastrectomy $(n=1)$, esophagectomy $(n=1)$, renal transplant $(n=1)$, and colectomy $(n=1)$. Most of the operations planned to use an open approach $(n=15)$, but several operations were to be carried out using laparoscopic methods $(n=6)$.

Records of patients undergoing the planned abdominal operation after TIPS were examined for perioperative complications, and these were then divided into those related to portal hypertension (ascites, variceal bleeding, etc.) and those that were unrelated. All of these perioperative complications were then included in this study.

The primary endpoint was whether patients underwent the planned abdominal operation after TIPS, with failure simply being defined as not proceeding to the planned abdominal operation. Reasons for failure to undergo the operation and outcomes of TIPS and abdominal operation were examined as secondary endpoints.

Numerical results included in the tables below follow the format: mean \pm standard deviation. Percentages, when relevant, are enclosed in parentheses.

\section{Results}

All 21 patients underwent TIPS creation as a preoperative measure. Patient characteristics prior to TIPS are shown in Table 1. Pressure measurements were recorded during the procedure for all but one patient (due to equipment failure). These values can be seen in Table 2. The mean portosystemic gradient prior to TIPS was $14.3 \mathrm{mmHg}$; this was reduced to a mean of $4.9 \mathrm{mmHg}$ after TIPS creation.

Hepatic encephalopathy was increased in the cohort following TIPS insertion, with 7 patients $(33.3 \%)$ experiencing new-onset symptoms within 30 days of the procedure. Thirtyday mortality after TIPS was $9.5 \%$. One patient died as a result of a transfusion related acute lung injury three days after TIPS. The second patient died 16 days after TIPS from sepsis secondary to complications of advanced sigmoid colon carcinoma. Neither death was directly attributable to the TIPS procedure itself.

After a median follow-up time of 705 days, 11 of the 21 patients who underwent TIPS creation had undergone the planned abdominal operation. Mean time between TIPS and operation was 38.7 days (range $=0-156$ days). There were no deaths within 30 days of the abdominal operation. One patient $(9.1 \%)$ had major perioperative morbidity related to portal hypertension and presented with wound dehiscence and infection (felt to be secondary to ascites) following hernia repair.

Ten of the original 21 patients had not undergone the planned abdominal operation by the median follow-up time of 705 days. Two of these patients died within 30 days of TIPS creation, as mentioned previously. Two other patients that had TIPS placement for ascites reduction prior to hernia repair did not proceed to the planned operation due to resolution of hernia symptoms after the ascites resolved. 
After TIPS, one patient had persistent hepatic encephalopathy requiring multiple hospitalizations. This required a downsize of the TIPS, which unfortunately lead to recurrence of the ascites. As a consequence, the patient was never able to be optimized for hernia repair.

Another patient was found to have multiple myeloma after TIPS creation and was no longer considered a candidate for the initially planned surgery. One patient lived several hours from the medical center and did not undergo operation due to documented transportation concerns. In three cases it was unknown why the patient failed to undergo the planned operation.

\section{Discussion}

Cirrhotic patients with portal hypertension present a unique challenge and often have comorbidities that complicate management. Operative intervention in this population has been associated with higher incidence of hemorrhage, wound dehiscence, infection, and renal dysfunction [9]. TIPS creation has been used as a method to improve surgical candidacy via portal decompression but data regarding the risks and benefits of that intervention are limited.

Only a few studies have examined the effects of preoperative TIPS placement. Vinet et al. found no benefit to preoperative TIPS placement when comparing a group of 18 patients who underwent TIPS to a group of 17 matched controls. It is possible that this was an anomaly, however, because the patients undergoing TIPS were generally more ill and had higher baseline Child-Pugh scores [7]. Fares et al. indicated a benefit with preoperative TIPS placement in a retrospective study involving 28 patients. Of the 28 patients with dedicated preoperative TIPS placement, 24 were able to undergo the planned operation with a thirtyday mortality of $0 \%$ and a one-year mortality of $22 \%$ [6].

Eleven of the 21 patients (52.4\%) in this study proceeded to the planned abdominal operation after undergoing preoperative TIPS creation. This is lower than the completion rate observed by Fares et al. (86\%) but this could be explained by differences in the patient population. Our study included a significant number of patients with non-alcoholic steatohepatitis (NASH) as the cause of liver disease, while the vast majority (93\%) of patients in the Fares et al. study had liver disease related to alcohol use or viral hepatitis [6]. Since NASH has a strong association with obesity, type 2 diabetes mellitus, hypertension, and hyperlipidemia it is possible that our patient population was already less fit to undergo operation [10].

Currently, both Child-Pugh and MELD scores are used in the preoperative evaluation of cirrhotic patients, since they have been shown to predict operative mortality. One frequently cited statistic regarding Child-Pugh scores is that patients in classes A, B, and C have operative mortality rates of $10 \%, 30 \%$, and $76 \%$, respectively, when undergoing major abdominal operation [11]. Although these figures have withstood the test of time and are consistent across studies, they are not particularly descriptive since each Child-Pugh class encompasses several different scores. The mean Child-Pugh score for patients undergoing 
abdominal operation in our cohort was 7.3, which is included in the range for Child-Pugh class B (scores of 7-9). However, it is unlikely that the operative mortality in these patients would be predicted to be as high as $30 \%$, since 7.3 represents the low side of that range. MELD scores are another important predictor of 30-day operative mortality in cirrhotic patients. In a large retrospective study, MELD score of 8-11 predicted a 30-day operative mortality rate of $10.3 \%$, while scores of $12-15$ predicted a 30 -day operative mortality rate of $25.4 \%$ [12]. When applying these rules to a group of patients, one encounters the same difficulties that occur with using Child-Pugh classes to predict operative mortality; namely that these percentages describe ranges and not individual scores. A simple heuristic described by Northup et. al is that each 1-point increase in the MELD score up to 20 points corresponds to a $1 \%$ increase in 30-day operative mortality rate [3]. For the patients in our cohort that underwent abdominal operation, the mean MELD score was 11.7, which would predict a 30-day operative mortality rate of approximately $11.7 \%$.

One of the most common complications following TIPS creation is the development of hepatic encephalopathy (HE). The incidence of HE after TIPS is reported to be between 25$45 \%$, although if only new and worsening cases of $\mathrm{HE}$ are considered this range drops to $13-36 \%$ [13]. Since many patients with severe liver disease have some symptoms of encephalopathy at baseline, this can be a difficult problem to quantify. Within 30 days of TIPS creation, 7 patients (33.3\%) in this cohort experienced new-onset HE. Patients that developed HE more than thirty days after TIPS were not included in this calculation because of the difficulty in assessing whether the HE was due to TIPS creation or overall worsening of hepatic function. It is worth noting that only one patient in this cohort experienced severe, refractory HE that required TIPS downsizing. The remainder of the patients were able to be managed with medical therapy which largely consisted of lactulose, rifaximin, and zinc.

One of the 11 patients undergoing abdominal operation experienced grade IIIa postoperative complications related to portal hypertension as defined by the Clavien-Dindo classification system [14]. This patient originally underwent preoperative TIPS to decrease ascites prior to hernia repair but experienced recurrent ascites, wound dehiscence, and infection in the postoperative period. This was unexpected since this patient had a portosystemic gradient pressure of $3 \mathrm{mmHg}$ after TIPS. This eventually required drain placement and antibiotic therapy.

Reasons for failure to undergo the planned abdominal operation were diverse and multifactorial. While 10 of the 21 patients $(47.6 \%)$ did not undergo the planned abdominal operation, it is worth noting that two of these patients no longer required surgical intervention because of the TIPS creation. Both of these patients underwent TIPS creation in preparation for hernia operation and had resolution of their hernia symptoms due to the decrease in ascites following TIPS. Resolution of hernia symptoms following TIPS is a somewhat unexpected finding since hernia incarceration and complications have been a reported outcome of TIPS [15]. Because all of the patients underwent TIPS specifically to improve their candidacy for a planned operation, it was surprising that reasons for failure could not be found for three patients. Additionally, another patient did not undergo the planned operation due to concerns regarding transportation. These outcomes highlight both 
the difficulty and importance of selecting patients who are likely to complete and benefit from this two-step process.

The main limitation of this study is its retrospective nature. This limits the ability to collect a more robust data set to allow for more in-depth analysis. Another limitation is the relatively small size of the series with only 22 patients undergoing preoperative TIPS creation during this time frame. Lastly, the authors recognize that a comparative arm of patients who underwent surgery without TIPS creation would be ideal. However, the patients in this series were not surgical candidates prior to the TIPS creation so no such comparative arm exists as all patients with these demographics required TIPS creation prior to operation.

\section{Conclusion}

In our population, TIPS creation allowed successful abdominal operation in the majority of patients, with thirty-day post-TIPS mortality of $9.5 \%$, no thirty-day operative mortality, and $9.1 \%$ major postoperative morbidity related to portal hypertension.

\section{Acknowledgements}

This study was funded, in part, by Award Number T35HL110854 from the National Institutes of Health. Special thanks to the Department of Radiology and Imaging Sciences at Indiana University School of Medicine for aiding in the development of this study.

Support

NIH T35 Research Fellowship

Previous Presentations

This paper was presented in abstract form at the Society of Interventional Radiology 2019 Annual Scientific Meeting, Austin, TX March 252019

\section{References}

1. Loffroy R, Favelier S, Pottecher P, Estivalet L, Genson PY, Gehin S, et al. Transjugular intrahepatic portosystemic shunt for acute variceal gastrointestinal bleeding: Indications, techniques and outcomes. Diagnostic and interventional imaging. 2015;96(7-8):745-55. [PubMed: 26094039]

2. de Goede B, Klitsie PJ, Lange JF, Metselaar HJ, Kazemier G. Morbidity and mortality related to non-hepatic surgery in patients with liver cirrhosis: a systematic review. Best practice \& research Clinical gastroenterology. 2012;26(1):47-59. [PubMed: 22482525]

3. Northup PG, Wanamaker RC, Lee VD, Adams RB, Berg CL. Model for End-Stage Liver Disease (MELD) predicts nontransplant surgical mortality in patients with cirrhosis. Annals of surgery. 2005;242(2):244-51. [PubMed: 16041215]

4. De Stefano F, Garcia CR, Gupta M, Marti F, Turcios L, Dugan A, et al. Outcomes in patients with portal hypertension undergoing gastrointestinal surgery: A propensity score matched analysis from the NSQIP dataset. The American Journal of Surgery. 2018.

5. Azoulay D, Buabse F, Damiano I, Smail A, Ichai P, Dannaoui M, et al. Neoadjuvant transjugular intrahepatic portosystemic shunt: a solution for extrahepatic abdominal operation in cirrhotic patients with severe portal hypertension. Journal of the American College of Surgeons. 2001;193(1):46-51. [PubMed: 11442253]

6. Fares N, Robic MA, Peron JM, Muscari F, Otal P, Suc B, et al. Transjugular intrahepatic portosystemic shunt placement before abdominal intervention in cirrhotic patients with portal hypertension: lessons from a pilot study. European journal of gastroenterology \& hepatology. 2018;30(1):21-6. [PubMed: 29049129] 
7. Vinet E, Perreault P, Bouchard L, Bernard D, Wassef R, Richard C, et al. Transjugular intrahepatic portosystemic shunt before abdominal surgery in cirrhotic patients: a retrospective, comparative study. Canadian journal of gastroenterology = Journal canadien de gastroenterologie. 2006;20(6):401-4. [PubMed: 16779457]

8. Corey KE, Kaplan LM. Obesity and liver disease: the epidemic of the twenty-first century. Clinics in liver disease. 2014;18(1):1-18. [PubMed: 24274861]

9. Kim JJ, Dasika NL, Yu E, Fontana RJ. Cirrhotic patients with a transjugular intrahepatic portosystemic shunt undergoing major extrahepatic surgery. Journal of clinical gastroenterology. 2009;43(6):574-9. [PubMed: 19169145]

10. Wong RJ, Ahmed A. Obesity and non-alcoholic fatty liver disease: Disparate associations among Asian populations. World journal of hepatology. 2014;6(5):263-73. [PubMed: 24868320]

11. O'Leary JG, Yachimski PS, Friedman LS. Surgery in the patient with liver disease. Clinics in liver disease. 2009;13(2):211-31. [PubMed: 19442915]

12. Teh SH, Nagorney DM, Stevens SR, Offord KP, Therneau TM, Plevak DJ, et al. Risk Factors for Mortality After Surgery in Patients With Cirrhosis. Gastroenterology. 2007;132(4):1261-9. [PubMed: 17408652]

13. Riggio O, Nardelli S, Moscucci F, Pasquale C, Ridola L, Merli M. Hepatic encephalopathy after transjugular intrahepatic portosystemic shunt. Clinics in liver disease. 2012;16(1):133-46. [PubMed: 22321469]

14. Dindo D, Demartines N, Clavien PA. Classification of surgical complications: a new proposal with evaluation in a cohort of 6336 patients and results of a survey. Annals of surgery. 2004;240(2):205-13. [PubMed: 15273542]

15. Smith MT, Rase B, Woods A, Trotter J, Gipson M, Kondo K, et al. Risk of hernia incarceration following transjugular intrahepatic portosystemic shunt placement. Journal of vascular and interventional radiology : JVIR. 2014;25(1):58-62. [PubMed: 24269791] 
Table 1.

Patient characteristics prior to TIPS $(n=21)$

\begin{tabular}{|c|c|}
\hline Age in years & $56.4 \pm 8.8$ \\
\hline \multicolumn{2}{|l|}{ Sex } \\
\hline Male & $13(61.9)$ \\
\hline Female & $8(38.1)$ \\
\hline \multicolumn{2}{|l|}{ Liver disease etiology } \\
\hline Non-alcoholic steatohepatitis (NASH) & $9(42.8)$ \\
\hline Alcohol & $6(28.6)$ \\
\hline Hepatitis $\mathrm{C}$ virus & $4(19.0)$ \\
\hline Autoimmune hepatitis & $1(4.8)$ \\
\hline Primary biliary cirrhosis & $1(4.8)$ \\
\hline \multicolumn{2}{|l|}{ Child-Pugh class } \\
\hline A & $8(38.1)$ \\
\hline $\mathrm{B}$ & $12(57.1)$ \\
\hline $\mathrm{C}$ & $1(4.8)$ \\
\hline Child-Pugh score & $7.2 \pm 1.5$ \\
\hline MELD score & $12.0 \pm 3.7$ \\
\hline MELD-Na score & $11.9 \pm 4.3$ \\
\hline Varices & $18(85.7)$ \\
\hline History of variceal bleeding & $5(23.8)$ \\
\hline History of ascites & $13(61.9)$ \\
\hline Ascites present at time of TIPS & $11(52.4)$ \\
\hline History of encephalopathy & $7(33.3)$ \\
\hline Uncontrolled encephalopathy present at time of TIPS & $0(0.0)$ \\
\hline Beta-blocker in use & $9(42.9)$ \\
\hline WBC & $5.2 \pm 2.5$ \\
\hline Hemoglobin & $11.7 \pm 2.0$ \\
\hline Platelets & $126.5 \pm 70.5$ \\
\hline INR & $1.3 \pm 0.2$ \\
\hline Prothrombin time & $14.2 \pm 2.5$ \\
\hline Sodium & $135.9 \pm 3.0$ \\
\hline
\end{tabular}

J Gastrointest Surg. Author manuscript; available in PMC 2021 October 01. 


$\mid$\begin{tabular}{l|l} 
Creatinine & $1.4 \pm 1.6$ \\
\hline Total bilirubin & $1.0 \pm .6$ \\
\hline ALT & $24.1 \pm 11.0$ \\
\hline Alkaline phosphatase & $87.8 \pm 25.7$ \\
\hline Albumin & $3.7 \pm 1.1$
\end{tabular}


Table 2.

Outcomes of TIPS and pressure measurements in $\mathrm{mmHg}(\mathrm{n}=20)$

\begin{tabular}{|l|l|}
\hline $\begin{array}{l}\text { Pre-TIPS } \\
\text { Portosystemic gradient }\end{array}$ & $14.3 \pm 4.6$ \\
\hline $\begin{array}{l}\text { Post-TIPS } \\
\text { Portosystemic gradient }\end{array}$ & $4.9 \pm 1.7$ \\
\hline Hepatic encephalopathy (new-onset) & $7(33.3)$ \\
\hline 30-day mortality & $2(9.5)$ \\
\hline
\end{tabular}

